\title{
Viscosity regularization iterative methods and convergence analysis
}

\author{
Dongfeng Lij ${ }^{\mathrm{a}}$, Juan Zhao ${ }^{\mathrm{b}, *}$ \\ a School of Information Engineering, North China University of Water Resources and Electric Power, Zhengzhou 450011, China. \\ ${ }^{b}$ School of Mathematics and Statistics, North China University of Water Resources and Electric Power, Zhengzhou 450011, China.
}

Communicated by X. Qin

\begin{abstract}
In this paper, a Moudafi's type viscosity regularization iterative method is introduced and investigated for an m-accretive mapping and a nonexpansive mapping. Strong convergence of the regularization iterative method is obtained in the framework of real uniformly smooth Banach spaces. Some subresults are also provided as applications of the main results. (C) 2017 All rights reserved.
\end{abstract}

Keywords: Accretive mapping, regularization iteration, uniform smoothness, operator equation. 2010 MSC: 47H05, 65J15, 47N10.

\section{Introduction and preliminaries}

Let $E$ be a real Banach space and let $E^{*}$ be the dual space of $E$. Let $\langle\cdot, \cdot\rangle$ denote the pairing between $E$ and $\mathrm{E}^{*}$. The normalized duality mapping $\mathrm{J}: \mathrm{E} \rightarrow 2^{\mathrm{E}^{*}}$ is defined by

$$
J(x)=\left\{x^{*} \in E^{*}:\left\langle x, x^{*}\right\rangle=\left\|x^{*}\right\|^{2}=\|x\|^{2}\right\}, \quad \forall x \in E .
$$

$E$ is said to be strictly convex if and only if $\|x+y\|<2$ for all $\|x\|=\|y\|=1$ and $x \neq y$. E is said to be uniformly convex if and only if for each $t>0$, there is an $r_{E}>0$ such that for $x, y \in E$ with $\|x\| \leqslant 1,\|y\| \leqslant 1$ and $\|x-y\| \geqslant t, r_{E} \leqslant \frac{2-\|x+y\|}{2}$ holds. The modulus of convexity of $E$ is the function $\delta_{\mathrm{E}}(\epsilon):(0,2] \rightarrow[0,1]$ defined by $\delta_{\mathrm{E}}(\epsilon)=\inf \left\{\frac{2-\|x+\mathrm{t}\|}{2}:\|x\|=\|y\|=1,\|x-y\| \geqslant \epsilon\right\}$. Let $p>1$ and $r>0$ be two fixed real numbers. We have the fact that Banach space $E$ is uniformly convex if and only if there exists a strictly increasing continuous convex function $\varphi:[0, \infty) \rightarrow[0, \infty)$ with $\varphi(0)=0$ such that

$$
a\|x\|^{p}-\left(a^{p}(1-a)+(1-a)^{p} a\right) \varphi(\|x-y\|)+(1-a)\|y\|^{p} \geqslant\|a x+(1-a) y\|^{p}
$$

for all $x, y \in B_{r}(0):=\{x \in E:\|x\| \leqslant r\}$ and $a \in[0,1]$.

Let $\mathrm{U}_{\mathrm{E}}=\{\mathrm{x} \in \mathrm{E}:\|\mathrm{x}\|=1\}$. $\mathrm{E}$ is said to have a Gâteaux differentiable norm (or is said to be smooth) if and only if

$$
\lim _{t \rightarrow 0} \frac{\|x+t y\|-\|x\|}{t}
$$

exists for each $x, y \in U_{E}$. $E$ is said to have a uniformly Gâteaux differentiable norm if and only if for each

\footnotetext{
*Corresponding author

Email addresses: yslidf@yeah.net (Dongfeng Li), zhaojuanyu@126.com (Juan Zhao)
} 
$y \in U_{E}$, the limit is attained uniformly for all $x \in U_{E}$. $E$ is said to have a uniformly Fréchet differentiable norm (or is said to be uniformly smooth) if and only if the limit is attained uniformly for $x, y \in U_{E}$. $E$ is uniformly smooth if and only if duality map J is single-valued and norm-to-norm uniformly continuous on bounded sets of $E$. It is also known that if the norm of $E$ is uniformly Gâteaux differentiable, then duality mapping J is uniformly norm to weak* continuous on each bounded subset of $E$.

Let $D$ be a nonempty subset of set $C$. A mapping $Q_{D}: C \rightarrow D$ is said to be a contraction if $Q_{D}^{2}=Q_{D}$. It is called sunny if for each $x \in C$ and $t \in(0,1)$, we have

$$
\mathrm{Q}_{\mathrm{D}} \mathrm{x}=\mathrm{Q}_{\mathrm{D}}\left(\mathrm{t} \mathrm{x}+(1-\mathrm{t}) \mathrm{Q}_{\mathrm{D}} \mathrm{x}\right) \text {. }
$$

$Q_{D}$ is said to be a sunny nonexpansive retraction if $Q_{D}$ is sunny, nonexpansive and a contraction. $D$ is said to be a nonexpansive retract of $C$ if there exists a nonexpansive retraction from $C$ onto $D$. It is known that $Q_{C}$ is sunny nonexpansive if and only if

$$
\left\langle x-Q_{c} x, \mathfrak{J}_{q}\left(y-Q_{c} x\right)\right\rangle \leqslant 0
$$

for all $x \in E, y \in C$.

Let $E$ be a uniformly smooth Banach space and let $C$ be a nonempty subset of $E$. Let $Q_{C}: E \rightarrow C$ be a retraction and $J$ be the duality mapping on $E$. Then the following are equivalent (see [22]):

(1) $Q_{C}$ is sunny and nonexpansive;

(2) $\left\langle x-y, J\left(Q_{c} x-Q_{c} y\right)\right\rangle \geqslant\left\|Q_{c} x-Q_{c} y\right\|^{2}, \forall x, y \in E ;$

(3) $\left\langle x-Q_{C} x, J\left(y-Q_{C} x\right)\right\rangle \leqslant 0, \forall x \in E, y \in C$.

It is well-known that if $E$ is a Hilbert space, then sunny nonexpansive retraction $Q_{C}$ is coincident with the metric projection $\mathrm{P}_{\mathrm{C}}$.

Let $C$ be a nonempty closed and convex subset of $E$. Let $S: C \rightarrow C$ be a mapping. Recall that $S$ is said to be contractive if and only if there exists a constant $\alpha \in(0,1)$ such that

$$
\|S x-S y\| \leqslant \alpha\|x-y\|, \quad \forall x, y \in C .
$$

From the Banach contractive principal, we see that every contractive mapping has a unique fixed point and the Picard iterative algorithm is convergent for the class of mappings. We also say $S$ is an $\alpha$-contraction. Recall that $\mathrm{S}$ is said to be nonexpansive if and only if

$$
\|S x-S y\| \leqslant\|x-y\|, \quad \forall x, y \in C .
$$

We here remark that the fixed point set of the class of nonexpansive may be empty. From [13], we see that the fixed point set of nonexpansive mapping is not empty provided that subset $C$ is bounded closed and convex and the framework of the space is uniformly convex; see also [3] and the references therein. In this paper, we always use $\operatorname{Fix}(S)$ to denote the fixed point set of $S$. Recently, approximation techniques of nonexpansive mapping have been investigated for convex optimization problem, in particular, variational inequality and equilibrium problems; see $[9,10,16,18]$ and the references therein.

Recall that Mann iterative algorithm generates a sequence in the following manner

$$
x_{1} \in C, \quad x_{n+1}=\left(1-\alpha_{n}\right) x_{n}+\alpha_{n} S x_{n}, \quad \forall n \geqslant 1,
$$

where $\left\{\alpha_{n}\right\}$ is a real number sequence in $(0,1)$ and $S$ is a nonexpansive mapping. Reich [21] showed that sequence $\left\{x_{n}\right\}$ converges weakly to a fixed point of $S$ provided that the framework of the space is smooth and control sequence $\left\{\alpha_{n}\right\}$ satisfies some conditions; see [21] and the references therein.

To guarantee the strong convergence of iterative sequences without any compact assumptions, we can use contractions to approximate a nonexpansive mapping. Define a contraction $\mathrm{F}: \mathrm{C} \rightarrow \mathrm{C}$ by

$$
\mathrm{Fx}=\mathrm{ku}+(1-\kappa) S x, \quad \forall x \in \mathrm{C}, \mathrm{k} \in(0,1),
$$


where $u$ is a fixed element in $C$ and $S: C \rightarrow C$ is an $\alpha$-contraction. Let $x_{k}$ denote the unique fixed point of $F$, that is, $x_{k}=k u+(1-k) S x_{k}$. From Browder [4], we see that $x_{k}$ converges strongly to a fixed point of $S$ as $\mathrm{K} \rightarrow 0$. Let $\mathrm{g}: \mathrm{C} \rightarrow \mathrm{C}$ be a contractive mapping and define a contraction $\mathrm{E}: \mathrm{C} \rightarrow \mathrm{C}$ by

$$
\mathrm{Ex}=\mathrm{kg}(\mathrm{x})+(1-\mathrm{k}) \mathrm{S} x, \quad \forall x \in \mathrm{C}, \mathrm{k} \in(0,1),
$$

where $S: C \rightarrow C$ is an $\alpha$-contraction. Let $x_{k}$ denote the unique fixed point of $E$, that is

$$
x_{\kappa}=\kappa g(\kappa)+(1-\kappa) S x_{\kappa} .
$$

Moudafi [15] proved that $x_{\kappa}$ converges strongly to a fixed point of $S$ as $\kappa \rightarrow 0$ and the fixed point is also a unique solution of some monotone variational inequality; see $[8,15,19]$ and the references therein.

Let Id denote the identity operator on $E$. An operator $A \subset E \times E$ with range

$$
\operatorname{Ran}(A)=\cup\{A z: z \in D(A)\},
$$

and domain $\operatorname{Dom}(A)=\{z \in E: A z \neq \emptyset\}$ is said to be accretive if for each $x_{i} \in D(A)$ and $y_{i} \in A x_{i}, i=1,2$, there exists $j\left(x_{1}-x_{2}\right) \in J\left(x_{1}-x_{2}\right)$ such that

$$
\left\langle y_{1}-y_{2}, j\left(x_{1}-x_{2}\right)\right\rangle \geqslant 0 .
$$

Interest in accretive operators stems mainly from their firm connection with equations of evolution and generalized variational inequalities; see $[1,6,11,17]$ and the references therein. From the viewpoint of geometry, accretive operator $A \subset E \times E$ has the following properties: the range of accretive operator $I+\lambda A$ increases, that is, $I+\lambda A$ is expansive. An accretive operator $A$ is said to be $m$-accretive if $\operatorname{Ran}(I+r A)=E$ for all $r>0$. In a real Hilbert space, an operator $A$ is $m$-accretive if and only if $A$ is maximal monotone. In this paper, we use $A^{-1}(0)$ to denote the set of zeros of $A$. For an accretive operator $A$, we can define a nonexpansive mapping $(\operatorname{Id}+r A)^{-1}: \operatorname{Ran}(I+r A) \rightarrow \operatorname{Ddom}(A)$, which is called the resolvent of $A$.

Fixed point theory of nonexpansive mappings has been applied to the zero point problem of accretive operators. One of the most popular techniques goes back to the work of Browder [4]. The basic idea is to use the resolvent operator. Rockafellar [23] introduced an iterative algorithm and called it the proximal point algorithm, which is now recognized as the Rockafellar's proximal point algorithm. He proved the weak convergence of the algorithm.

Recently, Kim and $\mathrm{Xu}$ [12], in the framework of real uniformly smooth Banach spaces, studied the following iterative algorithm

$$
\left\{\begin{array}{l}
x_{0} \in C \\
y_{n}=\left(I d+r_{n} A\right)^{-1} x_{n}, \\
x_{n+1}=\alpha_{n} u+\left(1-\alpha_{n}\right) y_{n}, \quad \forall n \geqslant 0
\end{array}\right.
$$

where Id is the identity operator, $u$ is a fixed element in $C$. They proved that sequence $\left\{x_{n}\right\}$ converges strongly to a zero point of $A$ provided that the control sequence $\left\{\alpha_{n}\right\}$ and $\left\{r_{n}\right\}$ satisfy some conditions; see [12] and the references therein. Recently, zero point problems of accretive operators via fixed point methods have been extensively investigated; see $[5,12,20,19]$ and the references therein.

Recently, Chang et al. [8] studies the following iterative scheme for accretive and nonexpansive operators via a viscosity approximate method:

$$
\left\{\begin{array}{l}
x_{0} \in C \\
y_{n}=\beta_{n} x_{n}+\left(1-\beta_{n}\right) S(I d+r A)^{-1} x_{n} \\
x_{n+1}=\alpha_{n} f\left(x_{n}\right)+\left(1-\alpha_{n}\right) y_{n}, \quad \forall n \geqslant 0
\end{array}\right.
$$

where $r$ is a positive real number sequence, Id is the identity operator, $\left\{\alpha_{n}\right\}$ and $\left\{\beta_{n}\right\}$ are two real number sequences in $(0,1), f$ is a contraction, $S$ is a nonexpansive mapping and $A$ is an accretive operator. Under 
some suitable restrictions imposed on the above sequences, they obtained a strong convergence theorem of common solution to problems of $S x=x$ and $A x=0$; see [8] and the references therein.

In this paper, motivated by the above results, we introduce and investigate a new Moudafi's type viscosity regularization iterative method and prove a strong convergence theorem.

Lemma 1.1 ([7]). In a Banach space $\mathrm{E}$, there holds the inequality

$$
\|x+y\|^{2} \leqslant\|x\|^{2}+2\langle y, j(x+y)\rangle, \quad \forall x, y \in E,
$$

where $j(x+y) \in J(x+y)$.

Lemma 1.2 ([14]). Let $\left\{a_{n}\right\},\left\{b_{n}\right\}$ and $\left\{c_{n}\right\}$ be three nonnegative real sequences satisfying

$$
a_{n+1} \leqslant\left(1-t_{n}\right) a_{n}+b_{n}, \quad \forall n \geqslant 0,
$$

where $\left\{t_{n}\right\}$ is a sequence in $(0,1)$. Assume that the following conditions are satisfied

$$
\sum_{n=0}^{\infty} t_{n}=\infty, \quad \limsup _{n \rightarrow \infty} \frac{b_{n}}{t_{n}} \leqslant 0 .
$$

Then $\lim _{n \rightarrow \infty} a_{n}=0$.

Lemma 1.3 ([24]). Let $\left\{x_{n}\right\}$ and $\left\{y_{n}\right\}$ be bounded sequences in a Banach space $E$ and $\left\{\beta_{n}\right\}$ be a sequence in $[0,1]$ with

$$
0<\liminf _{n \rightarrow \infty} \beta_{n} \leqslant \limsup _{n \rightarrow \infty} \beta_{n}<1 .
$$

Suppose that $x_{n+1}=\left(1-\beta_{n}\right) y_{n}+\beta_{n} x_{n}$, for all $n \geqslant 1$ and

$$
\limsup _{n \rightarrow \infty}\left(\left\|y_{n+1}-y_{n}\right\|-\left\|x_{n+1}-x_{n}\right\|\right) \leqslant 0
$$

Then $\lim _{n \rightarrow \infty}\left\|y_{n}-x_{n}\right\|=0$.

Lemma 1.4 ([2]). Let $\mathrm{E}$ be a Banach space and let $\mathrm{A}$ be an $\mathrm{m}$-accretive operator. For $\lambda>0$ and $\mu>0$ and $\mathrm{x} \in \mathrm{E}$, we have

$$
\mathrm{J}_{\lambda} x=\mathrm{J}_{\mu}\left(\frac{\mu}{\lambda} x+\left(1-\frac{\mu}{\lambda}\right) \mathrm{J}_{\lambda} x\right)
$$

where $\mathrm{J}_{\lambda}=(\mathrm{I}+\lambda \mathrm{A})^{-1}$ and $\mathrm{J}_{\mu}=(\mathrm{I}+\mu \mathrm{A})^{-1}$.

Lemma 1.5 ([19]). Let $\mathrm{E}$ be a uniformly smooth Banach space and let $\mathrm{C}$ be a nonempty closed convex subset of $\mathrm{E}$. Let $\mathrm{g}: \mathrm{C} \rightarrow \mathrm{C}$ be a fixed contraction and let $\mathrm{S}: \mathrm{C} \rightarrow \mathrm{C}$ be a nonexpansive mapping with a fixed point. Let $\left\{\mathrm{x}_{\mathrm{K}}\right\}$ be a sequence defined as follows

$$
x_{\kappa}=\kappa g\left(x_{\kappa}\right)+(1-\kappa) S x_{k}, \quad \forall \kappa \in(0,1) .
$$

Then $\left\{x_{\kappa}\right\}$ converges strongly as $\mathrm{k} \rightarrow 0$ to a fixed point $x^{*}$ of $S$, which is the unique solution in $\operatorname{Fix}(S)$ to the following variational inequality

$$
\left\langle x^{*}-g\left(x^{*}\right), J\left(x^{*}-p\right)\right\rangle \leqslant 0, \quad \forall p \in \operatorname{Fix}(S) .
$$

\section{Main results}

Theorem 2.1. Let $\mathrm{A}$ be an $\mathrm{m}$-accretive operators on a real uniformly smooth Banach space $\mathrm{E}$. Assume that $A^{-1}(0) \neq \emptyset$ and $\mathrm{C}:=\overline{\mathrm{D}(\mathrm{A})}$ is a convex subset of $\mathrm{E}$. Let $\mathrm{S}: \mathrm{C} \rightarrow \mathrm{C}$ be a nonexpansive mapping. Let $\mathrm{Q}_{\mathrm{C}}$ be the sunny nonexpansive retraction from $E$ onto $C$. Let $\left\{\alpha_{n}\right\},\left\{\beta_{n}\right\},\left\{\gamma_{n}\right\},\left\{\delta_{n}\right\},\left\{\lambda_{n}\right\}$, and $\left\{\omega_{n}\right\}$ be real number sequences in $(0,1)$. Let $\mathrm{g}: \mathrm{C} \rightarrow \mathrm{C}$ be a contractive mapping with constant $\alpha \in(0,1)$. Let $\left\{\mathrm{x}_{\mathrm{n}}\right\}$ be a sequence 
defined by

$$
\left\{\begin{array}{l}
x_{0} \in C, \\
x_{n+1}=\alpha_{n} g\left(x_{n}\right)+\beta_{n} x_{n}+\gamma_{n} y_{n}, \quad \forall n \geqslant 0, \\
y_{n}=\delta_{n} x_{n}+\lambda_{n} S \rho_{n}+\omega_{n} Q_{c} e_{n}, \quad \forall n \geqslant 0, \\
\rho_{n}=\left(I d+r_{n} A\right)^{-1} x_{n}, \quad \forall n \geqslant 0,
\end{array}\right.
$$

where $\left\{e_{n}\right\}$ is a bounded sequence in $E,\left\{r_{n}\right\}$ is a nonnegative real numbers sequence. Assume that $\left\{\alpha_{n}\right\},\left\{\beta_{n}\right\},\left\{\gamma_{n}\right\}$, $\left\{\delta_{n}\right\},\left\{\lambda_{n}\right\}$, and $\left\{\omega_{n}\right\}$ satisfy the following restrictions:

(a) $\alpha_{n}+\beta_{n}+\gamma_{n}=\delta_{n}+\lambda_{n}+\omega_{n}=1$, for each $n \geqslant 0$;

(b) $\lim _{n \rightarrow \infty} \alpha_{n}=0$ and $\sum_{n=1}^{\infty} \alpha_{n}=\infty$;

(c) $0<\liminf _{n \rightarrow \infty} \beta_{n} \leqslant \limsup _{n \rightarrow \infty} \beta_{n}<1$;

(d) $\sum_{n=0}^{\infty} \omega_{n}<\infty, \limsup _{n \rightarrow \infty} \delta_{n}<1$;

(e) $\lim _{n \rightarrow \infty}\left|\delta_{n+1}-\delta_{n}\right|=0, \lim _{n \rightarrow \infty}\left|\lambda_{n+1}-\lambda_{n}\right|=0$;

(f) $\lim _{n \rightarrow \infty} r_{n}=r \in(0,+\infty)$.

If $\operatorname{Fix}\left(S(I d+r A)^{-1}\right)=\operatorname{Fix}(S) \cap A^{-1}(0) \neq \emptyset$, then $\left\{x_{n}\right\}$ converges strongly to a point $x^{*} \in A^{-1}(0) \cap \operatorname{Fix}(S)$, which is the unique solution to the following variational inequality

$$
\left\langle f\left(x^{*}\right)-x^{*}, x^{*}-x\right\rangle \geqslant 0, \quad \forall x \in A^{-1}(0) \cap \operatorname{Fix}(S) .
$$

Proof. Fixing $p \in \operatorname{Fix}(S) \cap A^{-1}(0)$, we find that

$$
\left(I d+r_{n} A\right)^{-1} p=p=S p .
$$

Hence, we have

$$
\begin{aligned}
\left\|y_{n}-p\right\| & \leqslant \delta_{n}\left\|x_{n}-p\right\|+\lambda_{n}\left\|S \rho_{n}-p\right\|+\omega_{n}\left\|Q_{C} e_{n}-p\right\| \\
& \leqslant \delta_{n}\left\|x_{n}-p\right\|+\lambda_{n}\left\|\rho_{n}-p\right\|+\omega_{n}\left\|e_{n}-p\right\| \\
& \leqslant\left(\delta_{n}+\lambda_{n}\right)\left\|x_{n}-p\right\|+\omega_{n}\left\|e_{n}-p\right\| \\
& \leqslant\left\|x_{n}-p\right\|+\omega_{n} M_{1},
\end{aligned}
$$

where $M_{1}=\sup \left\{\left\|e_{n}-p\right\|: n \geqslant 1\right\}$. It follows that

$$
\begin{aligned}
\left\|x_{n+1}-p\right\| & \leqslant \alpha_{n}\left\|g\left(x_{n}\right)-p\right\|+\beta_{n}\left\|x_{n}-p\right\|+\gamma_{n}\left\|y_{n}-p\right\| \\
& \leqslant \alpha_{n}\left\|g\left(x_{n}\right)-g(p)\right\|+\alpha_{n}\|p-g(p)\|+\left(\beta_{n}+\gamma_{n}\right)\left\|x_{n}-p\right\|+\gamma_{n} \omega_{n} M_{1} \\
& \leqslant \alpha_{n} \alpha\left\|x_{n}-p\right\|+\alpha_{n}\|p-g(p)\|+\left(1-\alpha_{n}\right)\left\|x_{n}-p\right\|+\gamma_{n} \omega_{n} M_{1} \\
& \leqslant\left(1-\alpha_{n}(1-\alpha)\right)\left\|x_{n}-p\right\|+\alpha_{n}(1-\alpha) \frac{\|p-g(p)\|}{1-\alpha}+\omega_{n} M_{1} \\
& \leqslant \max \left\{\left\|x_{n}-p\right\|, \frac{\|p-g(p)\|}{1-\alpha}\right\}+\omega_{n} M_{1} \\
& \vdots \\
& \leqslant \max \left\{\left\|x_{1}-p\right\|, \frac{\|p-g(p)\|}{1-\alpha}\right\}+M_{1} \sum_{i=1}^{\infty} \omega_{i} .
\end{aligned}
$$

This proves that $\left\{x_{n}\right\}$ is bounded, so is $\left\{y_{n}\right\}$.

Putting $z_{n}=\frac{x_{n+1}-\beta_{n} x_{n}}{1-\beta_{n}}$, we have 


$$
\begin{aligned}
z_{n+1}-z_{n} & =\frac{x_{n+2}-\beta_{n+1} x_{n+1}}{1-\beta_{n+1}}-\frac{x_{n+1}-\beta_{n} x_{n}}{1-\beta_{n}} \\
& =\frac{\alpha_{n+1} g\left(x_{n+1}\right)+\gamma_{n+1} y_{n+1}}{1-\beta_{n+1}}-\frac{\alpha_{n} g\left(x_{n}\right)+\gamma_{n} y_{n}}{1-\beta_{n}} \\
& =\frac{\alpha_{n+1}\left(g\left(x_{n+1}\right)-y_{n+1}\right)+\left(1-\beta_{n+1}\right) y_{n+1}}{1-\beta_{n+1}}-\frac{\alpha_{n}\left(g\left(x_{n}\right)-y_{n}\right)+\left(1-\beta_{n}\right) y_{n}}{1-\beta_{n}} \\
& =\frac{\alpha_{n+1}}{1-\beta_{n+1}}\left(g\left(x_{n+1}\right)-y_{n+1}\right)-\frac{\alpha_{n}}{1-\beta_{n}}\left(g\left(x_{n}\right)-y_{n}\right)+y_{n+1}-y_{n} .
\end{aligned}
$$

It follows that

$$
\left\|z_{n+1}-z_{n}\right\| \leqslant \frac{\alpha_{n+1}}{1-\beta_{n+1}}\left\|g\left(x_{n+1}\right)-y_{n+1}\right\|+\frac{\alpha_{n}}{1-\beta_{n}}\left\|g\left(x_{n}\right)-y_{n}\right\|+\left\|y_{n+1}-y_{n}\right\| .
$$

Note that

$$
\begin{aligned}
\left\|y_{n+1}-y_{n}\right\| \leqslant & \delta_{n+1}\left\|x_{n+1}-x_{n}\right\|+\lambda_{n+1}\left\|S\left(\operatorname{Id}+r_{n+1} A\right)^{-1} x_{n+1}-S\left(\operatorname{Id}+r_{n} A\right)^{-1} x_{n}\right\| \\
& +\omega_{n+1}\left\|Q_{C} e_{n+1}-Q_{C} e_{n}\right\|+\left|\delta_{n+1}-\delta_{n}\right|\left\|x_{n+1}\right\| \\
& +\left|\lambda_{n}-\lambda_{n+1}\right|\left\|S\left(\operatorname{Id}+r_{n} A\right)^{-1} x_{n}\right\|+\left|\omega_{n}-\omega_{n+1}\right|\left\|e_{n}\right\| \\
\leqslant & \delta_{n+1}\left\|x_{n+1}-x_{n}\right\|+\lambda_{n+1}\left\|\left(\operatorname{Id}+r_{n+1} A\right)^{-1} x_{n+1}-\left(\operatorname{Id}+r_{n} A\right)^{-1} x_{n}\right\| \\
& +\omega_{n+1}\left\|e_{n+1}-e_{n}\right\|+\left|\delta_{n+1}-\delta_{n}\right|\left\|x_{n+1}\right\| \\
& +\left|\lambda_{n}-\lambda_{n+1}\right|\left\|S\left(\operatorname{Id}+r_{n} A\right)^{-1} x_{n}\right\|+\left|\omega_{n}-\omega_{n+1}\right|\left\|e_{n}\right\| .
\end{aligned}
$$

On the other hand, we have

$$
\begin{aligned}
& \left\|\left(\operatorname{Id}+r_{n+1} A\right)^{-1} x_{n+1}-\left(\operatorname{Id}+r_{n} A\right)^{-1} x_{n}\right\| \\
& \quad=\left\|\left(\operatorname{Id}+r_{n} A\right)^{-1} x_{n}-\left(\operatorname{Id}+r_{n} A\right)^{-1}\left(\left(1-\frac{r_{n}}{r_{n+1}}\right)\left(\operatorname{Id}+r_{n+1} A\right)^{-1} x_{n+1}+\frac{r_{n}}{r_{n+1}} x_{n+1}\right)\right\| \\
& \quad \leqslant\left\|\frac{r_{n}}{r_{n+1}} x_{n+1}+\left(1-\frac{r_{n}}{r_{n+1}}\right)\left(I d+r_{n+1} A\right)^{-1} x_{n+1}-x_{n}\right\| \\
& \quad \leqslant\left\|\frac{r_{n+1}-r_{n}}{r_{n+1}}\left(\left(I d+r_{n+1} A\right)^{-1} x_{n+1}-x_{n}\right)+\frac{r_{n}}{r_{n+1}}\left(x_{n+1}-x_{n}\right)\right\| \\
& \quad=\left\|\frac{r_{n+1}-r_{n}}{r_{n+1}}\left(\left(\left(I d+r_{n+1} A\right)^{-1} x_{n+1}-x_{n}\right)-\left(x_{n+1}-x_{n}\right)\right)+\left(x_{n+1}-x_{n}\right)\right\| \\
& \quad \leqslant \frac{M_{2}}{r_{n+1}}\left|r_{n+1}-r_{n}\right|+\left\|x_{n+1}-x_{n}\right\|,
\end{aligned}
$$

where

$$
M_{2}=\sup \left\{\left\|\left(\left(\operatorname{Id}+r_{n+1} A\right)^{-1} x_{n+1}-x_{n}\right)-\left(x_{n+1}-x_{n}\right)\right\|: n \geqslant 1\right\} .
$$

From (2.2) and (2.3), we find that

$$
\begin{aligned}
\left\|y_{n+1}-y_{n}\right\| \leqslant & \delta_{n+1}\left\|x_{n+1}-x_{n}\right\|+\frac{M_{2}}{r_{n+1}}\left|r_{n+1}-r_{n}\right|+\lambda_{n+1}\left\|x_{n+1}-x_{n}\right\| \\
& +\omega_{n+1}\left\|e_{n+1}-e_{n}\right\|+\left|\delta_{n+1}-\delta_{n}\right|\left\|x_{n+1}\right\| \\
& +\left|\lambda_{n}-\lambda_{n+1}\right|\left\|S\left(I d+r_{n} A\right)^{-1} x_{n}\right\|+\left|\omega_{n}-\omega_{n+1}\right|\left\|e_{n}\right\| \\
\leqslant & \left\|x_{n+1}-x_{n}\right\|+\frac{M_{2}}{r_{n+1}}\left|r_{n+1}-r_{n}\right| \\
& +\omega_{n+1}\left\|e_{n+1}-e_{n}\right\|+\left|\delta_{n+1}-\delta_{n}\right|\left\|x_{n+1}\right\| \\
& +\left|\lambda_{n}-\lambda_{n+1}\right|\left\|S\left(I d+r_{n} A\right)^{-1} x_{n}\right\|+\left|\omega_{n}-\omega_{n+1}\right|\left\|e_{n}\right\| .
\end{aligned}
$$


Substituting (2.4) into (2.1), we arrive at

$$
\begin{aligned}
\left\|z_{n+1}-z_{n}\right\|-\left\|x_{n+1}-x_{n}\right\| \leqslant & \frac{\alpha_{n+1}}{1-\beta_{n+1}}\left\|g\left(x_{n+1}\right)-y_{n+1}\right\|+\frac{\alpha_{n}}{1-\beta_{n}}\left\|g\left(x_{n}\right)-y_{n}\right\|+\frac{M_{2}}{r_{n+1}}\left|r_{n+1}-r_{n}\right| \\
& +\omega_{n+1}\left\|e_{n+1}-e_{n}\right\|+\left|\delta_{n+1}-\delta_{n}\right|\left\|x_{n+1}\right\| \\
& +\left|\lambda_{n}-\lambda_{n+1}\right|\left\|S\left(I d+r_{n} A\right)^{-1} x_{n}\right\|+\left|\omega_{n}-\omega_{n+1}\right|\left\|e_{n}\right\| .
\end{aligned}
$$

It follows that

$$
\limsup _{n \rightarrow \infty}\left(\left\|z_{n+1}-z_{n}\right\|-\left\|x_{n}-x_{n+1}\right\|\right) \leqslant 0 .
$$

Using Lemma 1.3, the restriction imposed on $\left\{\beta_{n}\right\}$ and the definition of $\left\{z_{n}\right\}$, we arrive at

$$
\lim _{n \rightarrow \infty}\left\|x_{n}-x_{n+1}\right\|=0 .
$$

Note that

$$
\left\|y_{n}-x_{n}\right\| \leqslant\left\|x_{n+1}-x_{n}\right\|+\alpha_{n}\left\|g\left(x_{n}\right)-y_{n}\right\|+\beta_{n}\left\|x_{n}-y_{n}\right\|
$$

It follows that

$$
\left\|y_{n}-x_{n}\right\| \leqslant \frac{\left\|x_{n+1}-x_{n}\right\|}{1-\beta_{n}}+\frac{\alpha_{n}}{1-\beta_{n}}\left\|g\left(x_{n}\right)-y_{n}\right\| .
$$

Using (2.5) and the restriction imposed on $\left\{\alpha_{n}\right\}$ and $\left\{\beta_{n}\right\}$, we see that

$$
\lim _{n \rightarrow \infty}\left\|x_{n}-y_{n}\right\|=0 \text {. }
$$

Note that

$$
\begin{aligned}
& \left\|x_{n}-S\left(I d+r_{n} A\right)^{-1} x_{n}\right\| \\
& \quad \leqslant\left\|x_{n}-y_{n}\right\|+\delta_{n}\left\|x_{n}-S\left(I d+r_{n} A\right)^{-1} x_{n}\right\|+\omega_{n}\left\|Q_{c} e_{n}-S\left(\operatorname{Id}+r_{n} A\right)^{-1} x_{n}\right\| .
\end{aligned}
$$

This implies that

$$
\left\|x_{n}-S\left(I d+r_{n} A\right)^{-1} x_{n}\right\| \leqslant \frac{\left\|x_{n}-y_{n}\right\|}{1-\delta_{n}}+\frac{\omega_{n}}{1-\delta_{n}}\left\|Q_{C} e_{n}-S\left(I d+r_{n} A\right)^{-1} x_{n}\right\| .
$$

Since $\lim \sup _{n \rightarrow \infty} \delta_{n}<1$, we may assume, without loss of generality, that $\delta_{n}<\delta<1$ for all $n$. Using (2.6), we get that

$$
\lim _{n \rightarrow \infty}\left\|x_{n}-S \rho_{n}\right\|=0
$$

From Lemma 1.4, we obtain that

$$
\begin{aligned}
\left\|(\operatorname{Id}+r A)^{-1}\left(x_{n}\right)-\rho_{n}\right\| & =\left\|(\operatorname{Id}+r A)^{-1}\left(\frac{r}{r_{n}} x_{n}+\left(1-\frac{r}{r_{n}}\right) \rho_{n}\right)-(\operatorname{Id}+r A)^{-1} x_{n}\right\| \\
& \leqslant\left\|x_{n}-\left(\left(1-\frac{r}{r_{n}}\right)\left(\operatorname{Id}+r_{n} A\right)^{-1} x_{n}+\frac{r}{r_{n}} x_{n}\right)\right\| \\
& \leqslant\left|r_{n}-r\right| \frac{M_{3}}{r_{n}}
\end{aligned}
$$

where $M_{3}=\sup \left\{\left\|\rho_{n}-x_{n}\right\|: n \geqslant 1\right\}$. It follows that

$$
\lim _{n \rightarrow \infty}\left\|(\operatorname{Id}+r A)^{-1}\left(x_{n}\right)-\rho_{n}\right\|=0 .
$$

On the other hand, we have

$$
\begin{aligned}
\left\|x_{n}-S(I d+r A)^{-1}\left(x_{n}\right)\right\| & \leqslant\left\|x_{n}-S \rho_{n}\right\|+\left\|S \rho_{n}-S(I d+r A)^{-1}\left(x_{n}\right)\right\| \\
& \leqslant\left\|\rho_{n}-(I d+r A)^{-1}\left(x_{n}\right)\right\|+\left\|x_{n}-S \rho_{n}\right\| .
\end{aligned}
$$


Combining (2.7) with (2.8), we arrive at

$$
\lim _{n \rightarrow \infty}\left\|x_{n}-S(I d+r A)^{-1} x_{n}\right\|=0 .
$$

Let $\kappa$ be a real number in $(0,1)$ and define a mapping $\Upsilon$ by

$$
\Upsilon=(1-\kappa) S(I d+r A)^{-1}+\kappa g \text {. }
$$

It follows that

$$
\begin{gathered}
\left\|\left((1-\kappa) S(I d+r A)^{-1} x+\kappa g(x)\right)-\left((1-\kappa) S(I d+r A)^{-1} y+\kappa g(y)\right)\right\| \\
(1-\kappa)\left\|S(I d+r A)^{-1} x-S(I d+r A)^{-1} y\right\|+\kappa\|g(x)-g(y)\| \\
(1-\kappa)\left\|(I d+r A)^{-1} x-(I d+r A)^{-1} y\right\|+\kappa \alpha\|x-y\| \\
\leqslant(1-\alpha(1-\kappa))\|x-y\|, \quad \forall x, y \in C .
\end{gathered}
$$

This shows that $\Upsilon$ is $(1-\alpha(1-\kappa))$-contractive. From the Banach contractive principal, we find that it has a unique fixed point. Next we use $x_{k}$ to denote the unique fixed point of $\Upsilon$, that is,

$$
(1-\kappa) S(I d+r A)^{-1} x_{\kappa}+\kappa g\left(x_{\kappa}\right)=x_{\kappa}, \quad \forall \kappa \in(0,1) .
$$

From Lemma 1.5, we find that $x_{\kappa} \rightarrow \bar{x}$, as $K \rightarrow 0$, where $\bar{x}=P_{A^{-1}(0) \cap F(S)} g(\bar{x})$, that is, $\bar{x}$ is a unique solution of generality variational inequality

$$
\langle\bar{x}-g(\bar{x}), j(\bar{x}-y)\rangle \leqslant 0, \quad \forall y \in A^{-1}(0) \cap F(S) .
$$

Next, we prove

$$
\limsup _{n \rightarrow \infty}\left\langle g(\bar{x})-\bar{x}, J\left(x_{n}-\bar{x}\right)\right\rangle \leqslant 0
$$

Note that

$$
\begin{aligned}
\left\|x_{n}-x_{k}\right\|^{2}= & (1-\kappa)\left\langle S(I d+r A)^{-1} x_{k}-x_{n}, j\left(x_{k}-x_{n}\right)\right\rangle+\kappa\left\langle g\left(x_{k}\right)-x_{n}, j\left(x_{k}-x_{n}\right)\right\rangle \\
= & (1-\kappa)\left\langle S(I d+r A)^{-1} x_{n}-x_{n}, j\left(x_{k}-x_{n}\right)\right\rangle \\
& +(1-\kappa)\left\langle S(I d+r A)^{-1} x_{k}-S(I d+r A)^{-1} x_{n}, j\left(x_{k}-x_{n}\right)\right\rangle \\
& +\kappa\left\langle x_{k}-x_{n}, j\left(x_{k}-x_{n}\right)\right\rangle+k\left\langle g\left(x_{k}\right)-x_{k}, j\left(x_{k}-x_{n}\right)\right\rangle \\
\leqslant & (1-\kappa)\left(\left\|S(I d+r A)^{-1} x_{n}-x_{n}\right\|\left\|x_{k}-x_{n}\right\|+\left\|x_{k}-x_{n}\right\|^{2}\right) \\
& +\kappa\left\langle g\left(x_{k}\right)-x_{k}, J\left(x_{k}-x_{n}\right)\right\rangle+\kappa\left\|x_{k}-x_{n}\right\|^{2} \\
\leqslant & (1-\kappa)\left\|S(I d+r A)^{-1} x_{n}-x_{n}\right\|\left\|x_{k}-x_{n}\right\|+\left\|x_{n}-x_{k}\right\|^{2} \\
& -\kappa\left\langle x_{k}-g\left(x_{k}\right), J\left(x_{k}-x_{n}\right)\right\rangle, \quad \forall \kappa \in(0,1) .
\end{aligned}
$$

This implies that

$$
\left\langle x_{k}-g\left(x_{k}\right), J\left(x_{k}-x_{n}\right)\right\rangle \leqslant \frac{(1-\kappa)\left\|x_{k}-x_{n}\right\|}{\kappa}\left\|S(I d+r A)^{-1} x_{n}-x_{n}\right\|, \quad \forall \kappa \in(0,1) .
$$

In view of (2.9), we find that

$$
\limsup _{n \rightarrow \infty}\left\langle x_{k}-g\left(x_{k}\right), J\left(x_{k}-x_{n}\right)\right\rangle \leqslant 0 .
$$

Taking the limsup as $k \rightarrow 0$ and noticing the fact that the two limits are interchangeable due to the fact the duality map J is norm-to-norm uniformly continuous on bounded sets, we obtain that (2.10) holds.

Finally, we show that $\lim _{n \rightarrow \infty}\left\|x_{n}-\bar{x}\right\|=0$. Note that $\left\|\rho_{n}-\bar{x}\right\| \leqslant\left\|x_{n}-\bar{x}\right\|$. It follows that

$$
\begin{aligned}
\left\|y_{n}-\bar{x}\right\| & =\left\|\delta_{n}\left(x_{n}-\bar{x}\right)+\lambda_{n}\left(\rho_{n}-\bar{x}\right)+\omega_{n}\left(Q_{C} e_{n}-\bar{x}\right)\right\| \\
& \leqslant \delta_{n}\left\|x_{n}-\bar{x}\right\|+\lambda_{n}\left\|\rho_{n}-\bar{x}\right\|+\omega_{n}\left\|Q_{C} e_{n}-\bar{x}\right\| \\
& \leqslant\left(1-\omega_{n}\right)\left\|x_{n}-\bar{x}\right\|+\omega_{n}\left\|e_{n}-\bar{x}\right\| .
\end{aligned}
$$


This in turn implies from Lemma 1.1 that

$$
\begin{aligned}
\left\|x_{n+1}-\bar{x}\right\|^{2}= & \left\|\beta_{n}\left(x_{n}-\bar{x}\right)+\gamma_{n}\left(y_{n}-\bar{x}\right)+\alpha_{n}\left(g\left(x_{n}\right)-\bar{x}\right)\right\| \\
\leqslant & \left\|\beta_{n}\left(x_{n}-\bar{x}\right)+\gamma_{n}\left(y_{n}-\bar{x}\right)\right\|^{2}+2 \alpha_{n}\left\langle\left(g\left(x_{n}\right)-\bar{x}\right), J\left(x_{n+1}-\bar{x}\right)\right\rangle \\
\leqslant & \left(\beta_{n}\left\|x_{n}-\bar{x}\right\|+\gamma_{n}\left\|y_{n}-\bar{x}\right\|\right)^{2}+2 \alpha_{n}\left\langle\left(g\left(x_{n}\right)-\bar{x}\right), J\left(x_{n+1}-\bar{x}\right)\right\rangle \\
\leqslant & \left(1-\alpha_{n}\right)^{2}\left\|x_{n}-\bar{x}\right\|^{2}+2 \alpha_{n}\left\langle g\left(x_{n}\right)-g(\bar{x}), J\left(x_{n+1}-\bar{x}\right)\right\rangle \\
& +2 \alpha_{n}\left\langle g(\bar{x})-\bar{x}, J\left(x_{n+1}-\bar{x}\right)\right\rangle \\
\leqslant & \left(1-\alpha_{n}\right)^{2}\left\|x_{n}-\bar{x}\right\|^{2}+2 \alpha_{n} \alpha\left\|x_{n}-\bar{x}\right\|\left\|x_{n+1}-\bar{x}\right\| \\
& +2 \alpha_{n}\left\langle g(\bar{x})-\bar{x}, J\left(x_{n+1}-\bar{x}\right)\right\rangle \\
\leqslant & \left(1-\alpha_{n}\right)^{2}\left\|x_{n}-\bar{x}\right\|^{2}+\alpha_{n} \alpha\left(\left\|x_{n}-\bar{x}\right\|^{2}+\left\|x_{n+1}-\bar{x}\right\|^{2}\right) \\
& +2 \alpha_{n}\left\langle g(\bar{x})-\bar{x}, J\left(x_{n+1}-\bar{x}\right)\right\rangle .
\end{aligned}
$$

It follows that

$$
\begin{aligned}
\left\|x_{n+1}-\bar{x}\right\|^{2} & \leqslant \frac{1+\alpha_{n}^{2}-\alpha_{n}(2-\alpha)}{1-\alpha_{n} \alpha}\left\|x_{n}-\bar{x}\right\|^{2}+2 \frac{\alpha_{n}}{1-\alpha_{n} \alpha}\left\langle g(\bar{x})-\bar{x}, J\left(x_{n+1}-\bar{x}\right)\right\rangle \\
& \leqslant \frac{1-\alpha_{n}(2-\alpha)}{1-\alpha_{n} \alpha}\left\|x_{n}-\bar{x}\right\|^{2}+2 \frac{\alpha_{n}}{1-\alpha_{n} \alpha}\left\langle g(\bar{x})-\bar{x}, J\left(x_{n+1}-\bar{x}\right)\right\rangle+M_{4} \alpha_{n}^{2},
\end{aligned}
$$

where $M_{4}$ is an appropriate constant such that $M_{4} \geqslant \sup \left\{\frac{\left\|x_{n}-\bar{x}\right\|^{2}}{1-\alpha_{n} \alpha}: n \geqslant 1\right\}$. Using Lemma 1.2, we obtain the desired conclusion immediately.

From Theorem 2.1, we have the following results.

Corollary 2.2. Let $\mathrm{A}$ be an $\mathrm{m}$-accretive operators on a real uniformly smooth Banach space $\mathrm{E}$. Assume that $A^{-1}(0) \neq \emptyset$ and $C:=\overline{D(A)}$ is a convex subset of $E$. Let $Q_{C}$ be the sunny nonexpansive retraction from $E$ onto C. Let $\left\{\alpha_{n}\right\},\left\{\beta_{n}\right\},\left\{\gamma_{n}\right\},\left\{\delta_{n}\right\},\left\{\lambda_{n}\right\}$, and $\left\{\omega_{n}\right\}$ be real number sequences in $(0,1)$. Let $\mathrm{g}: \mathrm{C} \rightarrow \mathrm{C}$ be a contractive mapping with constant $\alpha \in(0,1)$. Let $\left\{x_{n}\right\}$ be a sequence defined by

$$
\left\{\begin{array}{l}
x_{0} \in C \\
x_{n+1}=\alpha_{n} g\left(x_{n}\right)+\beta_{n} x_{n}+\gamma_{n} y_{n}, \quad \forall n \geqslant 0, \\
y_{n}=\delta_{n} x_{n}+\lambda_{n} \rho_{n}+\omega_{n} Q_{C} e_{n}, \quad \forall n \geqslant 0, \\
\rho_{n}=\left(I d+r_{n} A\right)^{-1} x_{n}, \quad \forall n \geqslant 0,
\end{array}\right.
$$

where $\left\{e_{n}\right\}$ is a bounded sequence in $\mathrm{E},\left\{r_{n}\right\}$ is a nonnegative real numbers sequence. Assume that $\left\{\alpha_{n}\right\},\left\{\beta_{n}\right\},\left\{\gamma_{n}\right\}$, $\left\{\delta_{n}\right\},\left\{\lambda_{n}\right\}$, and $\left\{\omega_{n}\right\}$ satisfy the following restrictions:

(a) $\alpha_{n}+\beta_{n}+\gamma_{n}=\delta_{n}+\lambda_{n}+\omega_{n}=1$, for each $n \geqslant 0$;

(b) $\lim _{n \rightarrow \infty} \alpha_{n}=0$ and $\sum_{n=1}^{\infty} \alpha_{n}=\infty$;

(c) $0<\liminf _{n \rightarrow \infty} \beta_{n} \leqslant \limsup _{n \rightarrow \infty} \beta_{n}<1$;

(d) $\sum_{n=0}^{\infty} \omega_{n}<\infty, \limsup _{n \rightarrow \infty} \delta_{n}<1$;

(e) $\lim _{n \rightarrow \infty}\left|\delta_{n+1}-\delta_{n}\right|=0, \lim _{n \rightarrow \infty}\left|\lambda_{n+1}-\lambda_{n}\right|=0$;

(f) $\lim _{n \rightarrow \infty} r_{n}=r \in(0,+\infty)$.

Then $\left\{x_{n}\right\}$ converges strongly to a zero point $x^{*}$ of $A$, which is the unique solution to the following variational inequality

$$
\left\langle f\left(x^{*}\right)-x^{*}, x^{*}-x\right\rangle \geqslant 0, \quad \forall x \in A^{-1}(0) .
$$


Corollary 2.3. Let $\mathrm{E}$ be a real uniformly smooth Banach space. Let $\mathrm{S}: \mathrm{C} \rightarrow \mathrm{C}$ be a nonexpansive mapping with fixed points. Let $Q_{C}$ be the sunny nonexpansive retraction from $E$ onto $C$. Let $\left\{\alpha_{n}\right\},\left\{\beta_{n}\right\},\left\{\gamma_{n}\right\},\left\{\delta_{n}\right\},\left\{\lambda_{n}\right\}$, and $\left\{\omega_{n}\right\}$ be real number sequences in $(0,1)$. Let $\mathrm{g}: \mathrm{C} \rightarrow \mathrm{C}$ be a contractive mapping with constant $\alpha \in(0,1)$. Let $\left\{x_{n}\right\}$ be a sequence defined by

$$
\begin{cases}x_{0} \in C & \\ x_{n+1}=\alpha_{n} g\left(x_{n}\right)+\beta_{n} x_{n}+\gamma_{n} y_{n}, & \forall n \geqslant 0, \\ y_{n}=\delta_{n} x_{n}+\lambda_{n} S x_{n}+\omega_{n} Q_{C} e_{n}, & \forall n \geqslant 0,\end{cases}
$$

where $\left\{e_{n}\right\}$ is a bounded sequence in E. Assume that $\left\{\alpha_{n}\right\},\left\{\beta_{n}\right\},\left\{\gamma_{n}\right\},\left\{\delta_{n}\right\},\left\{\lambda_{n}\right\}$, and $\left\{\omega_{n}\right\}$ satisfy the following restrictions:

(a) $\alpha_{n}+\beta_{n}+\gamma_{n}=\delta_{n}+\lambda_{n}+\omega_{n}=1$, for each $n \geqslant 0$;

(b) $\lim _{n \rightarrow \infty} \alpha_{n}=0$ and $\sum_{n=1}^{\infty} \alpha_{n}=\infty$;

(c) $0<\liminf _{n \rightarrow \infty} \beta_{n} \leqslant \limsup _{n \rightarrow \infty} \beta_{n}<1$;

(d) $\sum_{n=0}^{\infty} \omega_{n}<\infty, \limsup _{n \rightarrow \infty} \delta_{n}<1$;

(e) $\lim _{n \rightarrow \infty}\left|\delta_{n+1}-\delta_{n}\right|=0, \lim _{n \rightarrow \infty}\left|\lambda_{n+1}-\lambda_{n}\right|=0$.

Then $\left\{x_{n}\right\}$ converges strongly to a fixed point $x^{*}$ of $S$, which is the unique solution to the following variational inequality

$$
\left\langle f\left(x^{*}\right)-x^{*}, x^{*}-x\right\rangle \geqslant 0, \quad \forall x \in \operatorname{Fix}(S) .
$$

Remark 2.4. Our main results mainly improves the corresponding in Chang et al. [8] and Kim and Xu [12]. The stepsize of $r_{n}$ is variable instead of a fixed constant in Chang et al. [8] although it has a strong restrictions $\lim _{n \rightarrow \infty} r_{n}=r \in(0,+\infty)$. The restrictions imposed on the control sequences are mild. It deserves to mention that the common solution is also a unique solution to another monotone variational inequality. In Qin et al. [19], a viscosity approximation with continuous bounded and strong pseudocontractions are studied for the class of continuous pseudocontractions. It is of interest to extend the main results of this article to the viscosity approximation with continuous bounded and strong pseudocontractions in a nonuniformly smooth Banach space.

\section{References}

[1] I. K. Argyros, S. George, S. M. Erappa, Expanding the applicability of the generalized Newton method for generalized equations, Commun. Optim. Theory, 2017 (2017), 12 pages. 1

[2] V. Barbu, Nonlinear semigroups and differential equations in Banach spaces, Translated from the Romanian, Editura Academiei Republicii Socialiste România, Bucharest; Noordhoff International Publishing, Leiden, (1976). 1.4

[3] F. E. Browder, Fixed-point theorems for noncompact mappings in Hilbert space, Proc. Nat. Acad. Sci. U.S.A., 53 (1965), 1272-1276. 1

[4] F. E. Browder, Existence and approximation of solutions of nonlinear variational inequalities, Proc. Nat. Acad. Sci. U.S.A., 56 (1966), 1080-1086. 1

[5] L.-C. Ceng, C.-F. Wen, Y.-H. Yao, Iteration approaches to hierarchical variational inequalities for infinite nonexpansive mappings and finding zero points of m-accretive operators, J. Nonlinear Var. Anal., 1 (2017), 213-235. 1

[6] O. Chadli, A. Koukkous, A. Saidi, Existence of anti-periodic solutions for nonlinear implicit evolution equations with time dependent pseudomonotone operators, J. Nonlinear Var. Aanl., 1 (2017), 71-88. 1

[7] S.-S. Chang, Some problems and results in the study of nonlinear analysis, Proceedings of the Second World Congress of Nonlinear Analysts, Part 7, Athens, (1996), Nonlinear Anal., 30 (1997), 4197-4208. 1.1

[8] S.-S. Chang, H. W. J. Lee, C. K. Chan, Strong convergence theorems by viscosity approximation methods for accretive mappings and nonexpansive mappings, J. Appl. Math. Inform., 27 (2009), 59-68. 1, 2.4

[9] S. Y. Cho, B. A. Bin Dehaish, X.-L. Qin, Weak convergence of a splitting algorithm in Hilbert spaces, J. Appl. Anal. Comput., 7 (2017), 427-438. 1

[10] S. Y. Cho, X.-L. Qin, L. Wang, Strong convergence of a splitting algorithm for treating monotone operators, Fixed Point Theory Appl., 2014 (2014), 15 pages. 1 
[11] T. Kato, Nonlinear semigroups and evolution equations, J. Math. Soc. Japan, 19 (1967), 508-520. 1

[12] T.-H. Kim, H.-K. Xu, Strong convergence of modified Mann iterations, Nonlinear Anal., 61 (2005), 51-60. 1, 2.4

[13] W. A. Kirk, A fixed point theorem for mappings which do not increase distances, Amer. Math. Monthly, 72 (1965), 1004-1006. 1

[14] L. S. Liu, Ishikawa and Mann iterative process with errors for nonlinear strongly accretive mappings in Banach spaces, J. Math. Anal. Appl., 194 (1995), 114-125. 1.2

[15] A. Moudafi, Viscosity approximation methods for fixed-points problems, J. Math. Anal. Appl., 241 (2000), 46-55. 1

[16] M. A. Noor, R. Kamal, K. I. Noor, General variational inclusions and dynamical systems, Commun. Optim. Theory, 2017 (2017), 18 pages. 1

[17] M. A. Noor, T. Rassias, Z.-Y. Huang, Three-step iterations for nonlinear accretive operator equations, J. Math. Anal. Appl., 274 (2002), 59-68. 1

[18] X.-L. Qin, S. Y. Cho, Convergence analysis of a monotone projection algorithm in reflexive Banach spaces, Acta Math. Sci. Ser. B Engl. Ed., 37 (2017), 488-502. 1

[19] X.-L. Qin, S. Y. Cho, L. Wang, Iterative algorithms with errors for zero points of m-accretive operators, Fixed Point Theory Appl., 2013 (2013), 17 pages. 1, 1.5, 2.4

[20] X.-L. Qin, J.-C. Yao, Projection splitting algorithms for nonself operators, J. Nonlinear Convex Anal., 18 (2017), $925-935$. 1

[21] S. Reich, Weak convergence theorems for nonexpansive mappings in Banach spaces, J. Math. Anal. Appl., 67 (1979), 274-276. 1

[22] S. Reich, Strong convergence theorems for resolvents of accretive operators in Banach spaces, J. Math. Anal. Appl., 75 (1980), 287-292 1

[23] R. T. Rockafellar, Augmented Lagrangians and applications of the proximal point algorithm in convex programming, Math. Oper. Res., 1 (1976), 97-116 1

[24] T. Suzuki, Strong convergence theorems for infinite families of nonexpansive mappings in general Banach spaces, Fixed Point Theory Appl., 2005 (2005), 103-123. 1.3 